\title{
Effects of Gas Sensor Location in Olfactory Assist Mask
}

\author{
Haruka Matsukura $^{1}$, Shougo Okuda ${ }^{2}$, Hiroshi Ishida $^{2}$ \\ 'Osaka University, Osaka, Japan, \\ 2 Tokyo University of Agriculture and Technology, Tokyo, Japan \\ haruka.matsu@sys.es.osaka-u.ac.jp
}

\begin{abstract}
:
Experimental investigations conducted to show the effects of gas sensor location in an olfactory assist mask system is reported. An olfactory assist mask is an olfactory amplifier, which consists of a gas sensor and an odor generator. The sensor measures the concentration variations of a gaseous chemical compound wafting in the air. Then, the odor generator presents the user with an odor with amplified intensity. Therefore, the user of the olfactory assist mask can detect dilute gas as if his/her olfactory sensitivity were enhanced. Here we show that the usability of the olfactory assist mask can be improved by changing the position of the gas sensor. An experimental result is presented to show that placing a gas sensor on a foot of a human subject has an effect similar to putting his/her nose close to the ground. A gas plume trailing along the ground can be detected while walking upright, like a dog sniffing its nose near the ground by crouching down. An experimental result is also reported to show that the fluctuations of the response of a metal-oxide gas sensor which is caused by strong wind can be effectively reduced by placing the gas sensor in a flow cell.
\end{abstract}

Key words: gas sensor, olfactory display, human augmentation, olfaction, olfactory assist mask

\section{Introduction}

An olfactory assist mask is a system that can virtually enhance the sensitivity of our olfactory sensing [1]. The user of this system wears a gas mask as shown in Fig. 1. The system measures the concentration variations of a faint odor wafting in the air around the user by using a metal-oxide gas sensor placed on a mask. Then, it presents an odor with amplified concentration variations in the gas mask. An odor generator device is placed in the backpack carried on the user's back. The odor vapor with an amplified concentration is generated from an odorant set in the odor generator device.

In our previous work, gas tracking experiments were conducted in an indoor environment. The subjects were told to track an airborne faint odor plume and find its source [1]. The results show that the olfactory assist mask can make tracking of odor much easier. The success rate of finding an odor source was significantly increased when the subjects wore the olfactory assist mask.

In the experiments, however, the odor source was placed at an elevated position to let the subjects search for the odor source while walking in a natural posture. When an odor source is placed directly on the floor, the released odor often trails along the floor. Therefore, the searcher needs to crouch down to bring his/her face (and the gas sensor) closer to the floor. However, our body is not designed to work for long in such a posture, whereas the body of a dog is much more suitable for keeping the position of the nose close to the ground.

Here we report the investigations conducted to see the effects of the sensor position in the

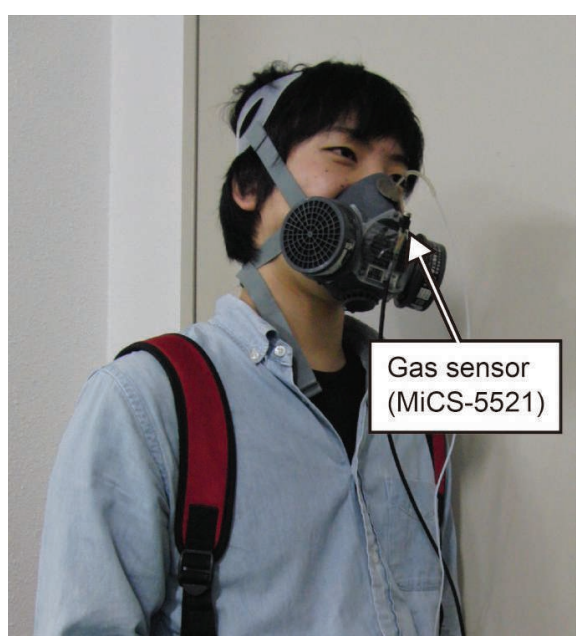

Fig. 1. Olfactory Assist Mask 


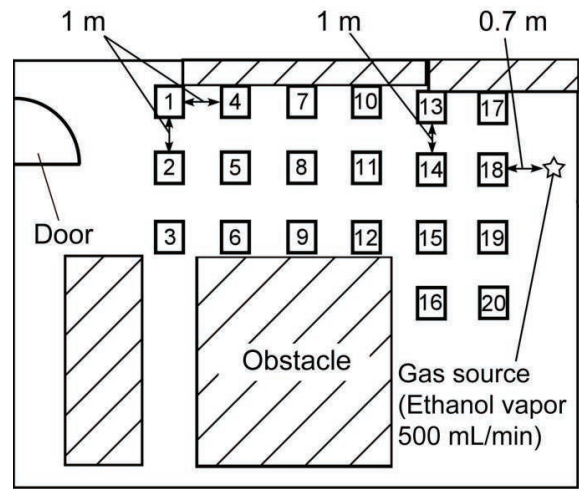

Fig. 2. Top view of the experimental setup. The measurement points were numbered from 1 to 20 .

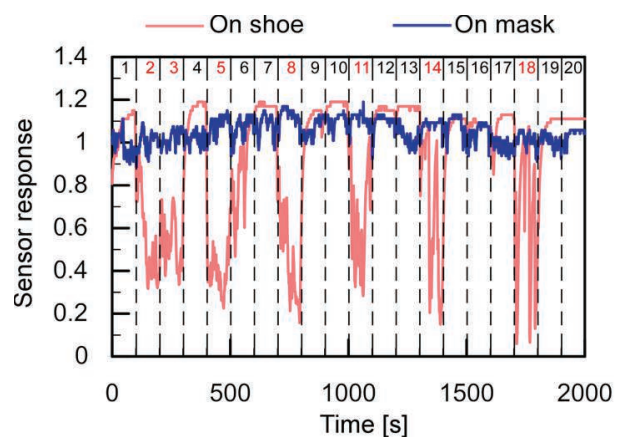

Fig. 3. Comparison of the response curves of the sensor placed on the shoe and the one on the mask.

olfactory assist mask system. The gas sensor was at first placed on the mask to minimize the distance from the user's nose. However, the sensor does not have to be there. The effect of placing the sensor in a flow cell is also discussed.

\section{Gas Plume Detection Experiments}

The experiments were conducted in a room shown in Fig. 2. A tube releasing saturated ethanol vapor at $500 \mathrm{~mL} / \mathrm{min}$ was placed on the right side of the room. The released gas was found to spread to the left side of the room along the floor owing to the weak airflow generated by the slight temperature differences in the room. To detect the gas plume trailing along the floor, the user of the olfactory assist mask needs to bring the gas sensor close to the floor. Therefore, we changed the position of the gas sensor.

The result is shown in Fig. 3 . The subject was asked to visit 20 measurement points in sequence and stops for $100 \mathrm{~s}$ at each measurement point. The response of the gas sensor (MiCS-5521, e2v technologies) shown in Fig. 2 was defined as the ratio of the sensor resistance in gas to that in air. Its value decreases when the gas concentration is increased. As shown in Fig. 2, almost no change in the sensor response was observed when the sensor was placed on the mask. When the

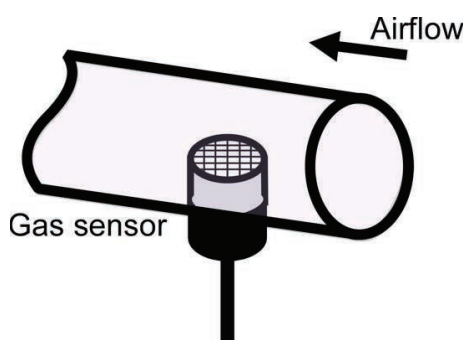

Fig. 4. Schematic diagram of sensor cell.

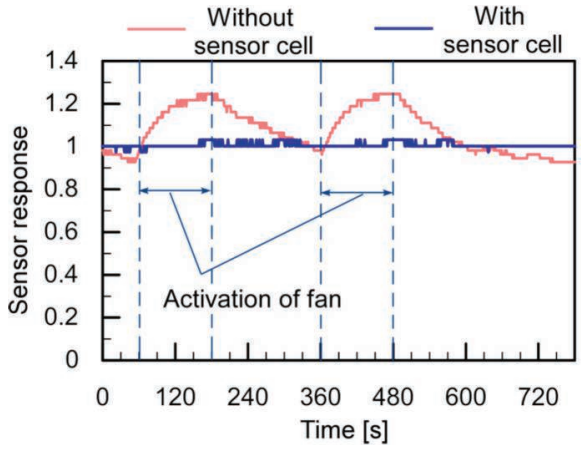

Fig. 5. Sensor response curves when exposed to wind.

sensor was placed on the shoe of the subject, gas with high concentration was detected at the positions downstream from the gas source.

\section{Placing Gas Sensor in Flow Cell}

The metal-oxide gas sensor in the olfactory assist mask has an internal heater. When the sensor is exposed to wind, its response value show fluctuations because the temperature of the sensing element is changed by the wind. To reduce the fluctuations, the sensor was placed in a plastic tube with a diameter of $7 \mathrm{~mm}$. Gas is introduced into this sensor cell by sucking air by a pump at a flow rate of $2 \mathrm{~L} / \mathrm{min}$. Fig. 5 shows the effect of the sensor cell. The sensor was exposed to airflow generated by an electric fan. Its velocity was $1.9 \mathrm{~m} / \mathrm{s}$. It can be seen that the change in the sensor response when exposed to the airflow was successfully suppressed.

\section{Conclusion}

The effects of gas sensor location in the olfactory assist mask were discussed. Placing the gas sensor on a show has an effect similar to virtually moving the nose on the foot. Placing the sensor in a flow cell effectively reduces the fluctuations of the gas sensor response caused by the wind. This work was supported in part by JSPS KAKENHI Grant Number 17K19964.

\section{References}

[1] H. Matsukura, H. Hashiguchi, H. Ishida, Tracking of a Gas Plume with the Aid of Olfactory Assist Mask, IEEE Sensors Journal 17, 5332-5340 (2017); doi: 10.1109/JSEN.2017.2721968 